\title{
Corneal epithelial changes following reconstruction by transplantation of limbal stem cells in goats with total limbal stem cell deficiency
}

\author{
Shengli Mi ${ }^{1}$, Zhongying Dou ${ }^{1}$, Qingmei Zhao ${ }^{1}$, Xueyi Yang ${ }^{1}$, Lei $\mathrm{Qu}^{1}$, Shuming Chen ${ }^{1}$, Jinlian Hua ${ }^{1}$ \\ ${ }^{l}$ Northwest university of A\&F, Shaanxi Branch of National Stem Cell Engineering and Technology Centre, Yangling, China
}

To study central and peripheral corneal epithelial changes after transplantation of limbal stem cells in goats with total limbal stem cell deficiency(LSCD).A state of total LSCD was induced in 15 goats. After transplantation with allograft of ex vivo expanded limbal epithelium on amniotic membrane (AM) on the eyes of 15 experimental goats, we surveiled the clinical outcomes of surface smoothness, stromal clarity, and vascularisation during the period of convalescence. Another 3 goats with total LSCD, only with injured tissue resected, were designed as negative control. Normal goat was also used as control. The hematoxylin and eosin staining was also used to evaluate the effects of the central and peripheral corneal epithelium. Immunofluorescence detection was performed with antibodies against CK3 keratin and P63 to assess the cell phenotype of central and peripheral corneal epithelium.

Within the first month following the surgery, there was a slight inflammatory reaction, the pupil and iris become unclear and the neo-blood vessels could be observed. After 2-6 months, the neo-blood gradually faded away. Over this period, the transplanted corneal stroma turned transparent again. The negative control eye was entirely covered with blood vessels and the cornea was completely opaque, completely lost vision. CK3 was expressed in all central and peripheral epithelial layers in normal and experimental corneal epithelium, was not found in the negative control corneal epithelium. $\mathrm{P}^{63}$ was also expressed in central and peripheral basal and suprabasal epithelia layer in normal and experimental corneal epithelium, and undetectable in negative control. Limbal stem cells associated with amniotic membrane transplantation are useful for restoring corneal epithelium phenotype in eyes with total LSCD. In contrast, the total LSCD model remains conjunctivalised corneas, which remain opaque with myofibroblasts.

Keywords: limbal stem cell, central, peripheral, tissue engineering

Cell Research (2008) 18:s72. doi: 10.1038/cr.2008.162; published online 4 August 2008

Correspondence: Shengli Mi ${ }^{\mathrm{a}}$, Zhongying Dou ${ }^{\mathrm{b}}$

a'E-mail: shenglimi@yahoo.com.cn

b-mail: douzhongying@china.com 